\title{
Forecasting Refrigerators Consumption to Support their Aggregated Participation in Demand Response
}

\author{
Pedro Faria, Aria Jozi, Zita Vale \\ Polytechnic of Porto \\ Porto, Portugal \\ \{pnf, arjoz, zav\}@isep.ipp.pt)
}

\begin{abstract}
Demand response programs have become very relevant. However, one of the important facts to have a reliable DR program is the creation of a clear and trustable perspective of the load consumption during the upcoming time periods. On another hand, the increment of the energy-based systems and different energy consuming appliances in the last decades results in larger daily energy consumption which creates the unpredictability of the energy demand. This variety of consumption profiles requires not only consideration of the total consumption of each consumer, but more detailed and focused studies on each type of energy consuming devices. Therefore, this paper proposes a system containing a combination of different forecasting and clustering algorithms to predict the power consumption of several refrigerators and aggregate them into certain groups based on the characteristics of their consumption profiles. Sequentially, the obtained results will be aggregated and serve as basis in order to schedule refrigerators in the context of a demand response program. In the case-study, 20000 refrigerators are considered.
\end{abstract}

Keywords- Aggregation, demand response, load prediction, refrigerator power consumption.

\section{INTRODUCTION}

The prediction models turned out to be one of the most focused and invested areas in past decades. This face has also influenced the energy and power consumption management system so that several studies have been published in this filed. Namely in [1], where a Fuzzy time series forecasting model has been used to predict the energy consumption. Or in [2] where several forecasting techniques based on different technologies such as Fuzzy Rule-Based Systems (FRBS), Support Vector Machines (SVM) and Artificial Neural Network (ANN) have been used to create a real-time energy consumption predictor for an office building.

The clustering techniques also are one of the most used analyses in the area of load and energy consumption, these techniques are widely used for consumer aggregations and load profiling. In [3] the actor uses the K-Means method to identify the daily load curves of a residential feeder, where the relationship between load profiles and seasonal periods is also considered and studied. Also in [4], two clustering algorithms namely as hierarchical and fuzzy c-means are used and compared in order to aggregate a group of electricity consumers and producers.

Demand Response (DR) programs refer to the change in the normal consumption pattern by end-consumers, in reaction to price signals or incentives provided by an aggregator, a system operator, or a market operator. The

This work has received funding from Portugal 2020 under SPEAR project (NORTE-01-0247-FEDER-040224) and from FEDER Funds through COMPETE program and from National Funds through (FCT) under the project UIDB/00760/2020, and CEECIND/02887/2017. provided consumption reduction will support the system operation when need, for example when a lack of supply or network constraint is verified. DR has been largely used in electricity markets, most of the times driven to large consumers.

In order to make small consumers able to participate in DR programs driven to large consumers, aggregators are needed [4]. An aggregator can collect the available demand response capacity and deliver it as a whole, to fit the DR program requirements. Also, the aggregator can focus on both generation and consumption resources, of several technologies and sizes.

In this paper, Wang and Mendel's Fuzzy Rule Learning Method and Support-vector machines are used to predict the load profile of a refrigerator during the next 24 hours. After determining the method with the most reliable results, it will be used to predict the load profile of 20000 refrigerators. The predicted profiles will then be aggregated into several groups using the K-Means clustering algorithm. Different number of clusters will be obtained in order to discuss the number of DR programs to be implemented. The characteristics of every cluster is analyzed and the cluster with most adaptive profiles for a DR program will be selected. The aggregator is able to request a demand reduction in the clusters that are more reliable to provide DR, considering their available consumption, one by one, until the desired demand reduction is reached. The strongness of this approach is that the forecasting and response deviations are grouped so a more reliable response to a DR event is obtained.

After this introduction section, the second section presents the details of the proposed model, including the implementation of the SVM, WM and K-Means methods. The case studies and the results of this work are presented in section three and finally, the fourth section includes the conclusions of this work.

\section{PROPOSED MODEL}

This paper uses a combination of forecasting and clustering techniques to divide a group of refrigerators into different clusters and use the most suitable cluster as the target of the DR event. The sequence of this proposed model has been presented in Figure 1 .

As can be seen in Figure 1, in the first step the historical data of the refrigerators will be used to create forecasting models to predict the profile of load consumption of every refrigerator during the upcoming 24 hours. In this phase, the system takes advantage of using two forecasting algorithms based on two different techniques. Namely as Support-vector machines [5], and Wang and Mendel model [6], which is a Fuzzy Rule-Based system [7]. These two forecasting methods have been chosen due to their reliability for load 
forecasting, according to the previous studies[8]. Based on the obtained results by these two forecasting methods for a single refrigerator, the method with the most exact values will be used to predict the load profile of a large number of refrigerators. For the next step, the predicted profiles must be divided into two different groups based on their characteristics. For this purpose, a clustering analysis using the K-means algorithm has been considered. In this process, the clustering method receives the predicted profile for every refrigerator and divides them into two several groups based on their similarities. The number of these groups can be between 5 to 10 related to the purpose of the DR program. In the final phase, one of the created clusters will be selected to be used as the target of the DR event due to the general characteristics of this cluster. Doing the forecast for one refrigerator, it is possible to assume that, as all the refrigerators will have similar behavior profiles, the best method for one refrigerator can be the best one for the other refrigerators also.

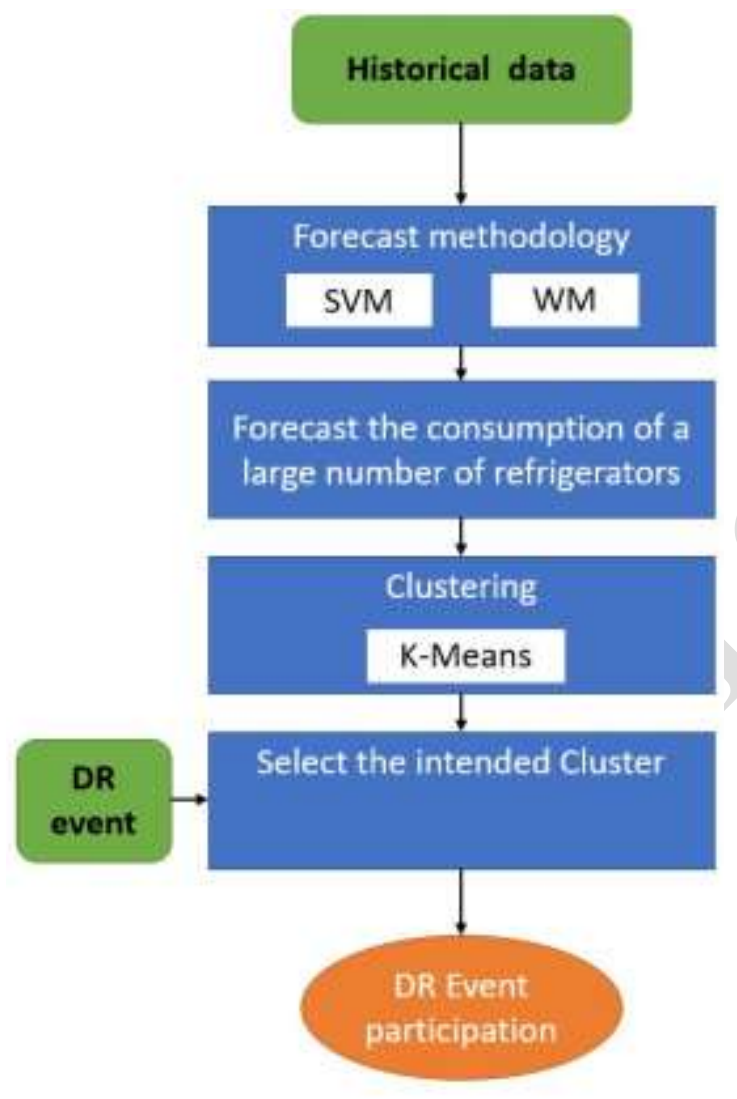

Fig. 1. The sequence of the proposed model

The following sections include the details of the implementations of the forecasting and clustering methods.

\section{A. Support-vector machines}

SVMs are one of the most used machine learning techniques to solve regression problems. SVM applications using Kernels are wildly used for reasons such as: often concentrating on convex problems; allowing many linear algebra techniques to be used in a non-linear way; have shown robustness in many application domains and spend fewer resources and half the time of artificial neural networks.

The first algorithm for pattern recognition was created in 1936, by R.A.Fisher. Further, the first running kernel of
SVM only for classification problems was implemented by a generalization of the nonlinear algorithm Generalized Portrait that has been created by Vapnik and Lerner in the sequence of [9]. The current SVM approach is implemented based on statistical learning theory [10] and the concept of the SVM can be tracked to the time that statistical learning theory was developed further with Vapnik, in 1979.

In this work, an R based library called "e 1071 " is used to implement the SVM method. This implantation can be used for bout classification and regression cases [11].

\section{B. Wang and Mendel's Fuzzy Rule Learning Method}

$\mathrm{WM}$ is a fuzzy rule-based learning algorithm that is one of the Fuzzy rule-based systems proposed in [12]. The fuzzy logic has been presented by Zadeh in 1965 [13] and this logic represents the reasoning of human experts in production rules (a set of IF-THEN rules) to handle real-life problems from domains such as control, prediction and inference, data mining, bioinformatics data processing, robotics, and speech recognition.

As having the required information in the right format from human experts can be difficult, acquiring the knowledge by generating the fuzzy IF-THEN rules automatically from the numerical training data can be an alternative and effective way. There are two critical processes that must be considered while modeling an FRBS, which are structure identification and parameter estimation. Structure identification is a process to find appropriate fuzzy IF-THEN rules and to determine the overall number of rules, and parameter estimation is applied to tune parameters of membership functions.

The WM model [6] has been widely known because of being simple and having a good performance [14]. The generation of the fuzzy rules bases is put into effect using four steps [15]:

1) Divide the Input and Output Spaces into Fuzzy Regions: This division might be obtained from the expert information that may exist or by a normalization process. symmetrical fuzzy partitions of triangular membership functions will be used in this order.

2) Generate Fuzzy Rules from Given Data Pairs: For the generation of the fuzzy rules, the train data will be used which is the output data from the first step.

3) Assign a degree to each rule: This step receives the created rules and determines a degree to each one. This degree is calculated by aggregating the degree of membership functions in the antecedent and consequent parts.

4) Create a Combined Fuzzy Rule Base: To provide the final rules, all the created rules will be divided into different groups and the rule with the highest importance degree is chosen in each group[16].

The WM algorithm in this work also has been implemented based on the $\mathrm{R}$ programming language by using the FRBS package[12].

\section{K-Means clustering algorithm}

The K-Means is a kind of clustering algorithm based on partition. K-means is the most used algorithm nowadays 
[17]. The fundamental idea of how K-means works is to verify a high intra-cluster similarity and a low inter-cluster similarity. The cluster's similarity is measured regarding the mean value of objects in the cluster. First, the user needs to select $\mathrm{K}$ initial clusters centers. The algorithm makes a random distribution of the data by the number of pre-defined clusters, calculating the intra-cluster, and inter-cluster measurements. The process repeats itself iteratively until an arrangement that meets the stop criterion is found, and the solution is returned [17]. The system uses the "cluster" library of $\mathrm{R}$ language [18]. This method is executed through a Java-based web server, developed for this purpose. This web service reserves the clustering input table in a JSON format and run the K-means in $\mathrm{R}$ using these data.

\section{Demand Response}

After performing the clustering of refrigerators, respecting their consumption profile, an aggregator can make use of the defined clusters to deploy DR events. The number of groups defined in the clustering process will correspond to the number of DR programs to be implemented. During the demand reduction of a specific event the set of clusters will be ordered according to their reduction capacity so that the aggregator can select one cluster after the other until the desired demand reduction is reached.

\section{RESULTS AND DISCUSSION}

This paper proposes a load consumption forecasting strategy based on using two forecasting algorithms namely as SVM and WM to forecast the load consumption of a refrigerator during a day by time intervals of 15 minutes. The method with the best performance will be selected to be used in order to predict the load consumption of a group of 20000 refrigerators. The consumption data has been obtained using a smart plug as explained in [19], in a refrigerator used in the authors' research center. In the future, with built in sensors, refrigerators are expected to provide the needed information. With the historical data for many months, it was possible to obtain and generate the daily profiles of 20000 refrigerators. In the sequence of this prediction, the predicted profiles will be aggregated to 10 groups by using a clustering analysis based on the K-Means algorithm. The cluster with the most suitable characteristics for a DR event will be chosen to be used as the target of the DR program.

\section{A. Load Forecasting}

The first step is to collect the train data for the forecasting methods. The train data in this study include the historical data of four variables which have the most influences on the load consumption of the refrigerators. These variables are humidity, the internal temperature of the refrigerator, the external temperature of the refrigerator, and the load consumption of past time periods. The methods receive this set of data from the past 3 mounts to be able to find the right pattern of the load consumption. After training the process every method predicts the load consumption of the refrigerator during the next 24 hours by time intervals of 15 minutes. Which means that every method provides 96 values as the result. the considered load value of every time period represents the maximum recorded load consumption of the refrigerator during these minutes.

Figure 2 presents the forecasted values by these two forecasting algorithms for the load consumption of a refrigerator in an office building for the 24 hours of an official day. The real values of every period are also included as well as the absolute error of each predicted value. As can be seen in this figure the intended refrigerator during a day has a load consumption between 0 to $120 \mathrm{~W}$ and also the predicted values do not overrun this interval. The most important challenge in this prediction is to be able to estimate correctly the pattern of the consumption and have the right perspective of the intervals which the refrigerator starts to consume. Based on the presented figure it can be concluded that both methods are able to predict these peak intervals of consumption by an acceptable error. However, to select the most trustable method the absolute error of each predicted value has been calculated and the method with the lower Mean Absolute Error (MAE) will be considered as the better method. MAPE index, using percentage errors, could also be considered. The absolute error addresses the absolute difference between the predicted and real values which in Figure 2 has been presented as SVM error and WM error.

The maximum absolute error of the SVM for these 96 forecasted values is $102.13 \mathrm{~W}$ and in the case of the WM is $120 \mathrm{~W}$. Both these values reference the periods that the methods are predicting that the refrigerator is turned on while it is not. On another hand, the MAE of the predicted values by SVM is $42.62 \mathrm{~W}$ while this value for $\mathrm{WM}$ is $22.54 \mathrm{~W}$ which makes the WM the more reliable forecasting method between these two in the case of this study. 


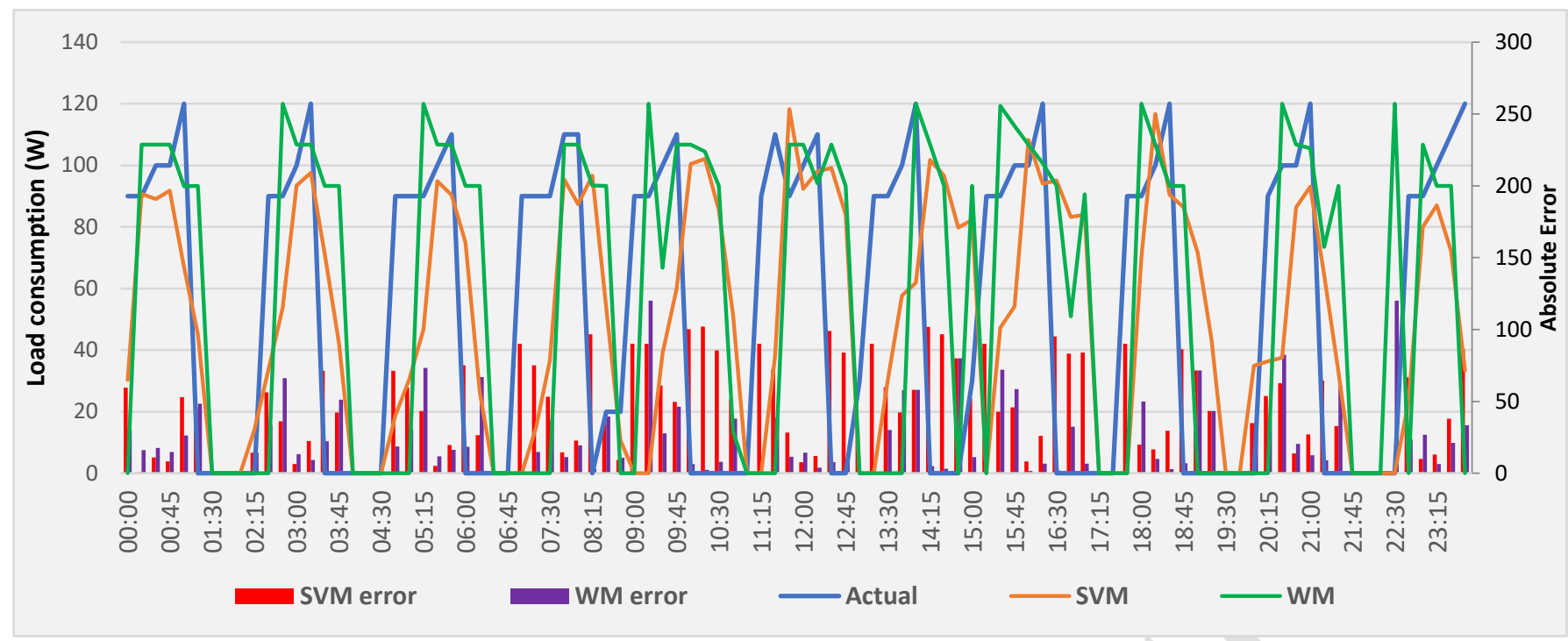

Fig. 2. The results of the refrigerator load consumption forecasting

This way, as the WM forecasting method has been recognized as the method with the most reliable predicted values, this method is used to predict the load consumption profile of 20000 refrigerators using the same strategy and same data sets as have been used in the case of the presented case study. In the following section, these predicted profiles will be aggregated through a clustering technique to be used for a DR program.

\section{B. Profile aggregation}

The objective of the aggregation process in this proposed model is to divide the refrigerators into a certain number of groups, due to the characteristics of the predicted load profile of each appliance. This way, the refrigerators with similar predicted load profiles during the day will be grouped and can be treated in the same way.

The number of the groups can be selected by the aggregator which for this study the considered number is 10. So, the aggregation process aims to divide the 20000 predicted load profiles into 10 clusters. As has been explained, the K-Means clustering algorithms have been chosen in this work to make this aggregation. This algorithm receives the 20000 load profiles plus the number of $\mathrm{K}$ which is 10 for this case. Figures 3 and 4 contain the results of this clustering analysis, where every line presents the average load profile of each cluster. As can been seen, every profile has different peak hours and variations along the day. Also, some clusters have a more symmetrical profile while some others have disordered patterns.

In order to evaluate the characteristics of every cluster two main factors have been considered: the average and Standard Deviation (SD) of load consumption during the day. The comparison of the average load consumption during the day helps to find the clusters with highest amount of consumption at the end of the day. Also, the SD of every cluster demonstrates that clusters have more peak values during the day. These characteristics can be used in order to recognize the best cluster for the DR program according to its objective. Table I presents the daily average and SD of every cluster consumption. Based on the presented results, the cluster 9 has the highest average followed by cluster 6 and 2 . This proves that these clusters have the highest amount of energy consumption at the end of the day. Also, the cluster 1 with the lowest average contains the refrigerators with the lowest energy consumption. Also, the SD values show that the cluster 10 has the highest variance followed by cluster 2 and 4 . The SD for cluster 9, which has the highest average, is 18.16 which is one of the lowest SDs. On another hand, the cluster 2 which has the third-highest average and the second-highest SD can be recognized as a profile with high consumption and high variance which can be used as the target of the DR programs. 


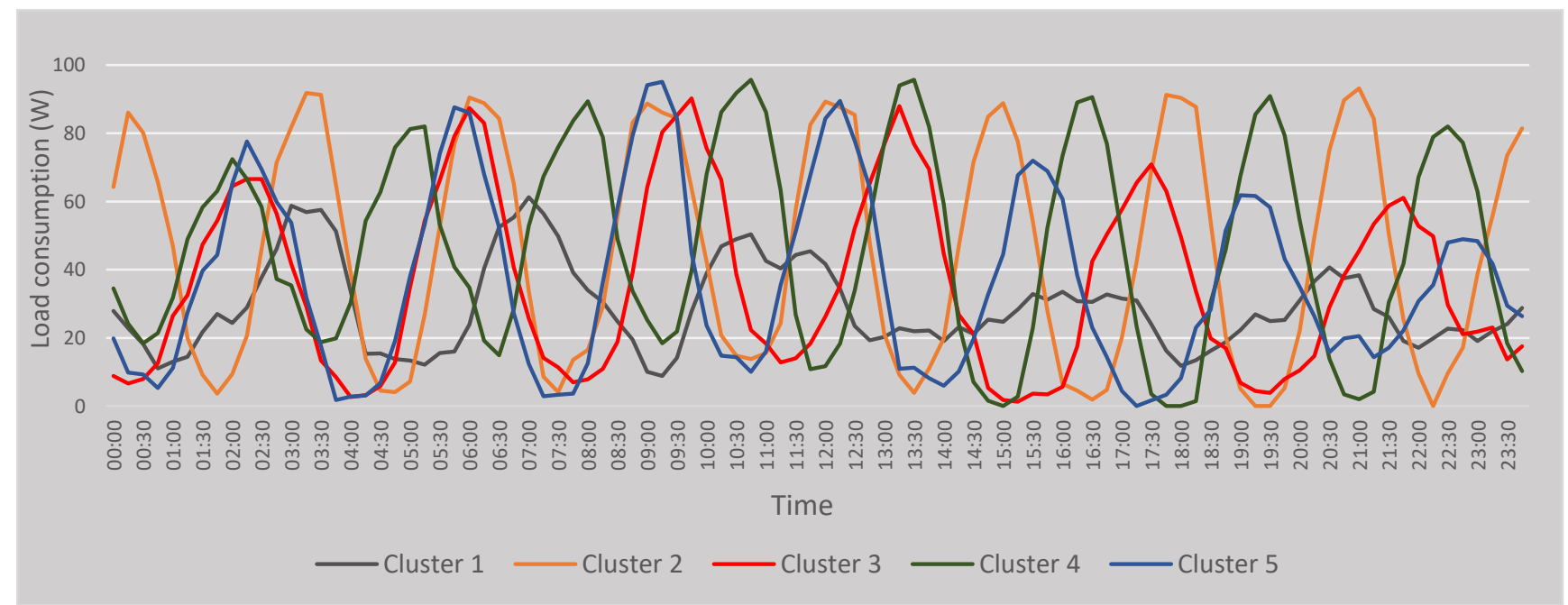

Fig. 3. Central load profiles of every cluster (1)

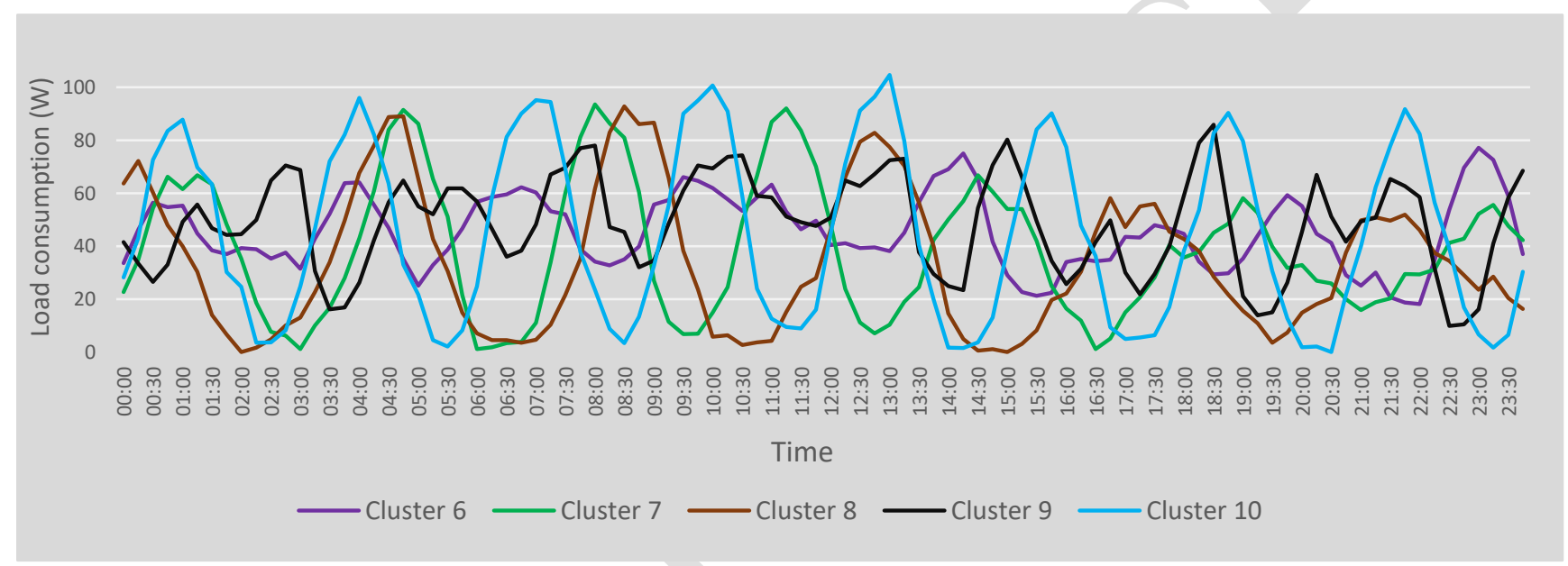

Fig. 4. Central load profiles of every cluster (2)

TABLE I. TABLE I - DAILY AVERAGE AND STANDARD DEVIATION CONSUMPTION OF EACH CLUSTER

\begin{tabular}{|c|c|c|}
\hline Cluster & Average & Standard Deviation \\
\hline 1 & 29.09926 & 12.76191681 \\
\hline 2 & 45.32708 & 32.30298631 \\
\hline 3 & 35.95219 & 25.93655641 \\
\hline 4 & 46.21474 & 29.02481832 \\
\hline 5 & 36.33712 & 26.43743562 \\
\hline 6 & 45.53023 & 13.71711249 \\
\hline 7 & 38.72653 & 24.99961909 \\
\hline 8 & 34.31906 & 26.18506806 \\
\hline 9 & $\mathbf{4 8 . 5 7 1 7 5}$ & 18.1627324 \\
\hline 10 & 44.54282 & $\mathbf{3 3 . 0 3 0 3 9 8 0 7}$ \\
\hline
\end{tabular}

One of the other important factors in a clustering process is the population of every cluster. The clusters with a higher number of refrigerators can be more ideal for a DR program due to a higher possibility of load reduction while the event includes more consumers. However, this can be not always true. In fact, it should be determined the average consumption per refrigerator in each cluster in order to determine the more relevant one to be firstly activated. In this study, the total number of consumers is 20000 and Table II includes the population of each cluster in case of a number between 3 to 10 as the $\mathrm{K}$.

As one can see in this table, in case that the number of clusters is 10 , the first cluster has the highest population by 3523 refrigerators, followed by the sixth cluster by 3446 . As the cluster 6 had also one of the highest consumption averages, this group is recognized as a group with a large number of refrigerators and high value of consumption, which is one of the most suitable clusters to be the target of the DR program. Also, the 1 cluster represents a group of refrigerators which includes the highest number of appliances with the lowest consumption.

TABLE II - POPULATION OF EACH CLUSTER IN THE CASE OF DIFFERENT NUMBERS OF CLUSTERS

\begin{tabular}{|c|c|c|c|c|c|c|c|c|}
\hline \multirow{2}{*}{ Cluster } & \multicolumn{7}{|c|}{ Number of clusters (k) } \\
\cline { 2 - 9 } & 3 & 4 & 5 & 6 & 7 & 8 & 9 & 10 \\
\hline $\mathbf{1}$ & 3731 & 6373 & 5467 & 2357 & 2150 & 1684 & 3576 & 3523 \\
\hline $\mathbf{2}$ & 8108 & 4767 & 3367 & 2254 & 4689 & 1580 & 2020 & 1269 \\
\hline $\mathbf{3}$ & 8161 & 5674 & 2383 & 2953 & 2642 & 1554 & 2487 & 2021 \\
\hline $\mathbf{4}$ & - & 3186 & 6244 & 4171 & 4275 & 1761 & 3058 & 1684 \\
\hline $\mathbf{5}$ & - & - & 2539 & 4224 & 1710 & 2203 & 1632 & 1425 \\
\hline $\mathbf{6}$ & - & - & - & 4041 & 2643 & 2850 & 1839 & 3446 \\
\hline
\end{tabular}




\begin{tabular}{|c|c|c|c|c|c|c|c|c|}
\hline $\mathbf{7}$ & - & - & - & - & 1891 & 4430 & 1606 & 1839 \\
\hline $\mathbf{8}$ & - & - & - & - & - & 3938 & 1761 & 1399 \\
\hline $\mathbf{9}$ & - & - & - & - & - & - & 2021 & 1995 \\
\hline $\mathbf{1 0}$ & - & - & - & - & - & - & - & 1399 \\
\hline
\end{tabular}

C. Forecast influence on the clustering results

As has been verified in previous sections, the accuracy of the forecasting methods can be different and related to the type of the methods and input data the predicted load profile can be more reliable. In this study, the SVM and WM forecasting methods were used to predict the load profile of a refrigerator during the upcoming 24 hours with intervals of 15 minutes. The forecasted values were analyzed and evaluated by being compared to the accrual values and as the conclusion, the WM method was selected as the most trustable method. In this section, the influence of the accuracy of the forecasted values on the results of clustering analyses will be verified. Here the same set of data as the last section is used (20000 predicted load profiles by WM) plus the predicted load profiles by SVM and WM as well as the actual values during the related day. The clustering process has been executed by different numbers of clusters $(\mathrm{K})$ namely as 3 to 10 . The results of this study are presented in Table III, where the associated number to each load profile in the case of every number of clusters are included.

TABLE III - ASSOCIATED CLUSTERS TO THE PREDICTED PROFILES IN THE CASE OF DIFFERENT NUMBERS OF CLUSTERS IN A REFRIGERATOR

\begin{tabular}{|c|c|c|c|}
\hline \multirow{2}{*}{ Number of clusters (K) } & \multicolumn{3}{|c|}{ Associated cluster } \\
\cline { 2 - 4 } & SVM & WM & Actual \\
\hline $\mathbf{3}$ & 2 & 2 & 2 \\
\hline $\mathbf{4}$ & 1 & 1 & 1 \\
\hline $\mathbf{5}$ & 5 & 5 & 5 \\
\hline $\mathbf{6}$ & 5 & 5 & 5 \\
\hline $\mathbf{7}$ & 6 & 6 & 6 \\
\hline $\mathbf{8}$ & 8 & 8 & 8 \\
\hline $\mathbf{9}$ & 7 & 2 & 2 \\
\hline $\mathbf{1 0}$ & 2 & 9 & 9 \\
\hline
\end{tabular}

As can be seen in this paper, while a lower number of $\mathrm{K}$ has been chosen, both predicted profiles by SVM and WM are associated with the same cluster as the actual profile. It is rational that while the number of $\mathrm{K}$ is lower the created clusters are less detailed and include a larger number of profiles. Although, when a larger number is selected as the number of $\mathrm{K}$, the accuracy of the predicted values makes a difference. Such as the case of 9 and 10 clusters that the predicted profile by WM is in the same cluster as the actual values, while the predicted profile by SVM is selected of another cluster.

\section{Demand Response implementation}

Looking at Table II, the aggregator managing DR programs is able to assign the number of clusters $k$ to the number of DR programs to be implemented. For example, if the aggregator wants to implement 3 DR programs, that can be one for each period of the day, then during the declaration of an event, each one of the 3 clusters are activated one after the other until the desired demand reduction is reached. In this example, the aggregator would activate cluster 3 as it has the largest number of refrigerators, then cluster 2 , and then cluster 1 , if needed. If the demand reduction provided by clusters 2 and 3 together is enough, cluster 1 is not activated.

Looking at Table III, it is possible to see that for a specific refrigerator, depending of the forecast method, different clusters can be assigned. This means that 2 refrigerators with similar characteristics can be allocated in different clusters due to the forecasted values. This is an important aspect to be accounted in the selection of the forecast method as it will impact on the fairness of demand response activation.

\section{CONCLUSION}

This paper proposed a combination of forecasting and clustering techniques to aggregate a large number of refrigerators in order to select the most adaptive group as the target of a DR program. Two forecasting methods, namely as SVM and WM were chosen to predict the load consumption of a refrigerator during the next 24 hours by a time interval of 15 minutes.

The evaluation of the forecasted result proved that the WM is a more trustable method due to the MAE errors of this method. Therefore, the WM was used to predict the load consumption of 20000 refrigerators in the same way. In the next step, these predicted profiles were aggregated by the KMean clustering method into several groups and the average consumption, Standard Deviation and the population of each group have been analyzed and the best group of refrigerators for the DR were recognized. Also, the influence of the accuracy of the forecasting results on the aggregation process has been studied and the importance of having more reliable predicted values has been proved.

\section{REFERENCES}

[1] V. Popov, M. Fedosenko, V. Tkachenko, and D. Yatsenko, "Forecasting Consumption of Electrical Energy Using Time Series Comprised of Uncertain Data", 2019 IEEE 6th International Conference on Energy Smart Systems (ESS), Kyiv, Ukraine, 2019, pp. 201-204, 2019.

[2] A. Jozi, D. Ramos, L. Gomes, P. Faria, T. Pinto, and Z. Vale, "Demonstration of an Energy Consumption Forecasting System for Energy Management in Buildings," In: Moura Oliveira P., Novais P., Reis L. (eds) Progress in Artificial Intelligence. EPIA 2019. Lecture Notes in Computer Science, vol 11804. Springer, Cham, pp. 462-468, 2019.

[3] S. A. Azad, A. B. M. S. Ali, and P. Wolfs, "Identification of typical load profiles using K-means clustering algorithm," Asia-Pacific World Congr. Comput. Sci. Eng. APWC CSE 2014, 2014.

[4] P. Faria, J. Spínola, and Z. Vale, "Aggregation and Remuneration of Electricity Consumers and Producers for the Definition of DemandResponse Programs," IEEE Trans. Ind. Informatics, vol. 12, no. 3, pp. 952-961, 2016.

[5] B. E. Boser, I. M. Guyon, and V. N. Vapnik, "A training algorithm for optimal margin classifiers," Proc. fifth Annu. Work. Comput. Learn. theory - COLT'92, pp. 144-152, 1992.

[6] L. X. Wang and J. M. Mendel, "Generating Fuzzy Rules by Learning from Examples," IEEE Trans. Syst. Man Cybern., vol. 22, no. 6, pp. 
1414-1427, 1992.

[7] L. S. Riza, C. Bergmeir, F. Herrera, and J. M. Benítez, "frbs : Fuzzy Rule-Based Systems for Classification and Regression in R," J. Stat. Softw., vol. 65, no. 6, 2015.

[8] A. Jozi, T. Pinto, I. Praça, and Z. Vale, "Day-ahead forecasting approach for energy consumption of an office building using support vector machines," in Proceedings of the 2018 IEEE Symposium Series on Computational Intelligence, SSCI 2018, 2019, pp. 1620-1625.

[9] A. L. V. Vapnik, "Pattern recognition using generalized portrait method," Autom. Remote Control, vol. 24, pp. 774-780, 1963.

[10] V. N. Vapnik, The Nature of Statistical Learning Theory. New york, 1995.

[11] D. Meyer, A. W. Evgenia Dimitriadou, Kurt Hornik, F. Leisch, C.-C. Chang, and C.-C. Lin, "Package "e1071,"” 2019.

[12] L. S. Riza, C. Bergmeir, F. Herrera, and J. M. Benítez, "\{frbs $\}$ : Fuzzy Rule-Based Systems for Classification and Regression in $\{\mathrm{R}\}, " R$ Packag. version 3.1-0, 2015.

[13] L. A. Zadeh and L. A, Fuzzy Sets. 1965.

[14] X. H. Yang, S. X. Guo, Y. H. Zhang, and H. T. Yang, "A sort of new energy consumption forecast method," Chinese Control Decis. Conf. 2008, CCDC 2008, pp. 3611-3615, 2008.

[15] F. Casillas, J.; Córdon, O.; Herrera, "Improving the Wang and Mendel' s Fuzzy Rule Learning Method by Inducing Cooperation Among Rules," 8th Inf. Process. Manag. Uncertain. KnowledgeBased Syst. Conf., pp. 1682-1688, 2000.

[16] A. Jozi, T. Pinto, I. Praça, and Z. Vale, "Decision Support Application for Energy Consumption Forecasting," Appl. Sci., vol. 9, no. 4, p. 699, 2019.

[17] D. $\mathrm{Xu}$ and Y. Tian, "A Comprehensive Survey of Clustering Algorithms," Ann. Data Sci., vol. 2, no. 2, pp. 165-193, Jun. 2015.

[18] M. Maechler, P. Rousseeuw, A. Struyf, and K. Hubert, M. Hornik, "cluster: Cluster Analysis Basics and Extensions. R package version 1.15.2.," p. 2014, 2014.

[19] L. Gomes, F. Sousa, Z. Vale, "An Intelligent Smart Plug with Shared Knowledge Capabilities.", Sensors 2018, 18, 3961. 